\title{
Subdivision of de novo metastatic nasopharyngeal carcinoma based on tumor burden and pretreatment Epstein-Barr virus DNA for therapeutic guidance of primary tumor radiotherapy
}

\author{
Jin-Hao Yang \\ Sun Yat-sen University Cancer Center \\ Xue-Song Sun \\ Sun Yat-sen University Cancer Center \\ Bei-Bei Xiao \\ Sun Yat-sen University Cancer Center \\ Li-Ting Liu \\ Sun Yat-sen University Cancer Center \\ Shan-Shan Guo \\ Sun Yat-sen University Cancer Center \\ Jia-dong Liang \\ First Affiliated Hospital of Sun Yat-sen University \\ Guo-Dong Jia \\ Sun Yat-sen University Cancer Center \\ Lin-Quan Tang \\ Sun Yat-sen University Cancer Center \\ Qiu-Yan Chen \\ Sun Yat-sen University Cancer Center \\ Hai-Qiang Mai ( $\nabla$ maihq@sysucc.org.cn ) \\ Sun Yat-sen University Cancer Center
}

\section{Research Article}

Keywords: nasopharyngeal carcinoma, distant metastasis, locoregional radiotherapy, EBV DNA, risk stratifications

Posted Date: February 19th, 2021

DOI: https://doi.org/10.21203/rs.3.rs-196099/v1

License: (c) (1) This work is licensed under a Creative Commons Attribution 4.0 International License. Read Full License 


\section{Abstract}

Purpose: To improve individualized treatment of de novo metastatic nasopharyngeal carcinoma (dmNPC) patients by investigating prognostic factors and identifying patients who achieved better survival outcomes after locoregional radiotherapy (LRRT).

Materials and methods: Our study included a cohort of 498 dmNPC patients. Overall survival (OS) was the primary endpoint. We analyzed the correlation of all potential prognostic factors and survival outcomes by Kaplan-Meier survival curves using log-rank test and Cox proportional hazards regression model.

Results: Multivariate analysis identified three independent prognostic factors: Epstein-Barr virus (EBV) DNA, number of metastatic lesions, and number of metastatic organs. Through these factors, we successfully divided all patients into 3 subgroups: low-risk (single metastatic organ, EBV DNA $\leq 25,000$ copies/ml, and $\leq 5$ metastatic lesions), intermediate-risk (single metastatic organ, EBV DNA > 25,000 copies/ml, and $\leq 5$ metastatic lesions), and high-risk (multiple metastatic organs or $>5$ metastatic lesions or both). By comparing LRRT and non-RT groups, we found statistical differences in OS in the low-risk and intermediate-risk subgroups $(p=0.039$ and $p=0.010$, respectively) but no significant difference in OS in the high-risk subgroup $(p=0.076)$. Further multivariate analysis of different risk stratifications revealed that LRRT was a protective factor only for the low- and intermediate-risk subgroups.

Conclusions: The risk stratification of dmNPC may be used as a new prognostic factor to help clinicians organize individualized LRRT treatment to improve the survival outcomes of dmNPC patients.

\section{Introduction}

Nasopharyngeal carcinoma (NPC) is a malignancy predominantly associated with infection by the Epstein-Barr virus (EBV) (1). It originates in the nasopharyngeal epithelium, which is found on the nasopharyngeal posterior wall. Approximately 12,900 new cases of NPC occur each year, with more than $70 \%$ of cases occurring in the east and southeast Asia $(1,2)$. Differently from ordinary head and neck squamous cell carcinoma, NPC is highly sensitive to radiotherapy (RT) due to its particular biological properties. With the development of RT technology, the 3-year local control rate and survival rates of non-metastatic NPC reached $80-90 \%$ in the intensitymodulated RT (IMRT) era (3). Notably, among new cases of NPC, $6-15 \%$ of patients are diagnosed with distant metastatic NPC (de novo mNPC, dmNPC) $(4,5)$. Whether these patients should receive locoregional RT (LRRT) needs to be clarified.

According to a previous study, dmNPC patients that underwent LRRT plus palliative chemotherapy (PCT) achieved greater overall survival (OS) than those that received PCT alone. However, it was not clear whether all patients benefited from the primary tumor treatment (6). In 2020, the National Comprehensive Cancer Network (NCCN) guidelines recommended LRRT alone following systemic chemotherapy for patients with oligometastatic disease (7). Nevertheless, the numbers of metastatic sites and organs that should be considered as "localized" or "widespread" have not been reported. Additionally, the aforementioned study did not analyze pretreatment plasma EBV DNA concentration, which is regarded as a prognosis indicator for NPC, to select candidates for LRRT (6).

Therefore, we conducted this study to investigate prognostic factors for dmNPC patients and to identify patients who achieved improved OS after LRRT by taking the prognostic factors into consideration. With our results, we aim to provide important information for the individualized treatment of these patients.

\section{Methods}

All methods were carried out in accordance with relevant guidelines and regulations as the National Comprehensive Cancer Network (NCCN) guidelines and eighth edition of the American Joint Committee on Cancer staging system. All patients in our study were staged by eighth edition of the American Joint Committee on Cancer staging system and all patients were treated according to the NCCN guidelines.

\section{Patients}

In total, 11,235 patients were newly diagnosed with NPC in the Sun Yat-sen University Cancer Center in China from November 2006 to October 2016. Of these, 498 patients were enrolled in this retrospective study (Fig. 1) according to the following inclusion criteria: (I) dmNPC patients diagnosed histologically or radiologically; (II) age between 18 and 70 years; (III) no history of malignancy or synchronous cancer; (IV) treatment with cisplatin-based chemotherapy regimen; (V) normal hematopoietic function: white blood cell count $\geq 4 \times 10^{9} / \mathrm{L}$, platelets $\geq 100 \times 10^{9} / \mathrm{L}$, hemoglobin $\geq 90 \mathrm{~g} / \mathrm{L}$, and neutrophil granulocytes $>2.0 \times 10 / \mathrm{L} ;(\mathrm{VI})$ normal liver function test: 
aspartate aminotransferase and alanine aminotransferase < 2.5-fold of upper limit of normal (ULN), and total bilirubin < 2.0×ULN; (VII) normal renal function test: creatinine clearance $\geq 60 \mathrm{ml} / \mathrm{min}$ or creatinine $\leq 1.5 \times \mathrm{ULN}$; and (VIII) male or non-pregnant female. All patients were restaged based on the 8th edition of the American Joint Committee on Cancer/International Union Against Cancer staging system. This study was approved by the clinical research ethics committee of Sun-Yat sen university cancer center, and written informed consent was obtained from each patient.

During enrollment, general evaluation tests mainly included physical examination of the head and neck region (including nasopharynx and cervical lymph nodes), physical examination of the nervous system, EBV serologic tests, EBV DNA quantitative determination, nasal endoscopy, lesion biopsy, head and neck magnetic resonance imaging (MRI) scan, emission compared tomography (ECT) scan, and chest and abdominal CT scan. Positron emission tomography (PET-CT) was considered an optional evaluation test based on the patient's financial burden.

We evaluated metastatic lesions and organs based on radiological criteria. The number of metastatic bone lesions and metastatic lesions (excluding bones) were assessed by ECT (or PET-CT) and chest and abdominal CT (or PET-CT) scans, respectively. The number of metastatic organs was evaluated by both ECT and chest and abdominal CT (or PET-CT) scans.

\section{Plasma EBV DNA essay}

Pretreatment plasma EBV DNA concentrations were measured using quantitative polymerase chain reaction. The detailed procedure is reported elsewhere (8).

\section{Treatment}

All included patients received cisplatin-based induction chemotherapy (IC). The common PCT regimens were as follows: (I) triplet docetaxel-cisplatin-fluorouracil: $60 \mathrm{mg} / \mathrm{m}^{2}$ of docetaxel and $60 \mathrm{mg} / \mathrm{m}^{2}$ of cisplatin on day 1 plus $500-800 \mathrm{mg} / \mathrm{m}^{2}$ of 5 -fluorouracil for 120 hours; (II) docetaxel-cisplatin: $75 \mathrm{mg} / \mathrm{m}^{2}$ of docetaxel on day 1 plus $20-25 \mathrm{mg} / \mathrm{m}^{2}$ of cisplatin on days $1-3$; (III) cisplatinfluorouracil: $20-25 \mathrm{mg} / \mathrm{m}^{2}$ of cisplatin on days $1-3$ plus $800-1000 \mathrm{mg} / \mathrm{m}^{2}$ of 5 - fluorouracil for 96 hours; (IV) gemcitabine-cisplatin: $1000 \mathrm{mg} / \mathrm{m}^{2}$ of gemcitabine on day 1 plus $20-25 \mathrm{mg} / \mathrm{m}^{2}$ of cisplatin on days $1-3$. Each regimen was administered intravenously every 3 weeks for a total of $4-6$ courses. Among the 498 dmNPC patients, 311 received local RT after IC with two-dimensional conventional radiotherapy or IMRT techniques. The total radiation doses were 68-70 Gy for nasopharyngeal and neck lesions, with a frequency of five fractions per week divided in 1.8-2.2 Gy fractions (9).

\section{Follow-up}

All patients underwent follow-up examinations every 3 months for the first 3 years and every 6 months thereafter. The examinations included EBV DNA copy detection, nasopharyngoscopy, head and neck MRI scan, chest and abdominal CT scan, and ECT or PET/CT scans. The primary endpoint of this study was OS, which was measured from initial diagnosis to death from any cause or loss to follow-up.

\section{Statistical analysis}

The clinical characteristics of patients from different treatment groups were compared using the Pearson $\chi^{2}$ test. The number of metastatic lesions and EBV DNA copies were transformed into dichotomous variables based on cutoff values defined by the receiveroperating characteristic (ROC) analysis. The survival outcomes of patients from different subgroups were analyzed using Kaplan-Meier curves and the log-rank test. The independent prognosis predictors were evaluated using the Cox proportional hazards regression model. All data analyses were performed using the Statistical Package for Social Sciences (SPSS for macOS, version 21.0, IBM Corp., Armonk, NY). A two-tailed $p<0.05$ was considered statistically significant.

\section{Results}

\section{Patient characteristics}

In total, $498 \mathrm{dmNPC}$ patients were included in this analysis. Among them, $378(75.3 \%)$ and $123(24.7 \%)$ patients had single and multiple metastatic organs, respectively. The median age was 47 years (range, $18-77$ years) and most patients were male $(83.1 \%, 414 / 498)$. Regarding metastatic lesions, $338(67.9 \%)$ and 160 (32.1\%) patients had five or less and more than five lesions, respectively. Based on ROC analysis, the EBV DNA copies cutoff value was set at 25,000 copies/ml and $284(57.0 \%)$ patients had levels that surpassed this value. As shown in Table 1, we found statistical differences in the number of metastatic organs, number of metastatic lesions, and pretreatment EBV DNA copies between the different treatment groups. 
Table 1

The clinical characteristics of the patients that did RT and did not do RT

\begin{tabular}{|c|c|c|c|c|}
\hline Characteristic & Total & non-RT & RT & $P$ value \\
\hline & $\mathrm{N}(\%)$ & $\mathrm{N}(\%)$ & $\mathrm{N}(\%)$ & \\
\hline \multicolumn{5}{|l|}{ Age (years) } \\
\hline$\leq 47$ & $246(49.4)$ & $87(46.5 \%)$ & $159(51.1 \%)$ & 0.355 \\
\hline$>47$ & $252(50.6)$ & $100(53.5 \%)$ & $152(48.9 \%)$ & \\
\hline \multicolumn{5}{|l|}{ Gender } \\
\hline Male & $414(83.1)$ & $155(82.9 \%)$ & $259(83.3 \%)$ & 1.000 \\
\hline Female & $84(498)$ & $32(17.1 \%)$ & $52(16.7 \%)$ & \\
\hline \multicolumn{5}{|l|}{ Smoking } \\
\hline No smoking & $278(55.8)$ & $109(58.3 \%)$ & $169(54.3 \%)$ & 0.403 \\
\hline Smoking & $220(44.2)$ & $78(41.7 \%)$ & $142(45.7 \%)$ & \\
\hline \multicolumn{5}{|l|}{ Family history } \\
\hline No & $447(89.8)$ & $170(90.9 \%)$ & $277(89.1 \%)$ & 0.545 \\
\hline Yes & $51(10.2)$ & $17(9.1 \%)$ & $34(10.9 \%)$ & \\
\hline \multicolumn{5}{|l|}{ T stage } \\
\hline $\mathrm{T}_{1}-\mathrm{T}_{2}$ & $83(16.7)$ & $29(15.5 \%)$ & $54(17.4 \%)$ & 0.621 \\
\hline $\mathrm{T}_{3}-\mathrm{T}_{4}$ & $415(83.3)$ & $158(84.5 \%)$ & $257(82.6 \%)$ & \\
\hline \multicolumn{5}{|l|}{ N stage } \\
\hline $\mathrm{N}_{0}-\mathrm{N}_{1}$ & $103(20.7)$ & $30(16.0 \%)$ & $73(23.5 \%)$ & 0.052 \\
\hline $\mathrm{N}_{2}-\mathrm{N}_{3}$ & 398 (79.3) & $157(84.0 \%)$ & $238(76.5 \%)$ & \\
\hline \multicolumn{5}{|c|}{ No. of metastatic organs } \\
\hline 1 & $378(75.3)$ & $116(62.0 \%)$ & $259(83.3 \%)$ & $<0.001$ \\
\hline$>1$ & $123(24.7)$ & $71(38.0 \%)$ & $52(16.7 \%)$ & \\
\hline \multicolumn{5}{|c|}{ No. of metastatic lesions } \\
\hline$\leq 5$ & $338(67.9)$ & $97(51.9 \%)$ & $241(77.5 \%)$ & $<0.001$ \\
\hline$>5$ & $160(32.1)$ & $90(48.1 \%)$ & $70(22.5 \%)$ & \\
\hline \multicolumn{5}{|c|}{ EBV-DNA(Copies/ml) } \\
\hline$\leq 25,000$ & $214(43.0)$ & $59(31.6 \%)$ & $155(49.8 \%)$ & $<0.001$ \\
\hline$>25,000$ & $284(57.0)$ & $128(68.4 \%)$ & $156(50.2 \%)$ & \\
\hline \multicolumn{5}{|c|}{ Chemotherapy regimens } \\
\hline TPF & $128(25.7)$ & $40(21.4 \%)$ & $88(28.3 \%)$ & 0.001 \\
\hline TP & $121(24.3)$ & $36(19.3 \%)$ & $85(27.3 \%)$ & \\
\hline PF & $129(25.9)$ & $58(31.0 \%)$ & $71(22.8 \%)$ & \\
\hline GP & $27(5.4)$ & $18(9.6 \%)$ & $9(2.9 \%)$ & \\
\hline others & $93(18.7)$ & $35(18.7 \%)$ & $58(18.6 \%)$ & \\
\hline
\end{tabular}




\section{Analysis of clinical characteristics' influences on the prognosis of dmNPC patients}

All factors that may influence prognosis were included in the Cox proportional hazards regression model. As shown in Table 2, we found a higher mortality risk for patients who had metastasis in multiple organs (hazard ratio [HR], 1.897; 95\% confidence interval [Cl], 1.4012.568; $p<0.001)$, more than five metastatic lesions ( $H R, 2.246 ; 95 \% \mathrm{Cl}, 1.670-3.020 ; p<0.001)$, or pretreatment EBV DNA concentrations above 25,000 copies/ml (HR, 1.479; $95 \% \mathrm{Cl}, 1.132-1.930 ; \mathrm{p}=0.004)$, whereas patients who underwent RT had a lower risk of death $(\mathrm{HR}, 0.665 ; 95 \% \mathrm{Cl}, 0.511-0.864 ; \mathrm{p}=0.002)$. Thus, we concluded that multiple organs metastasis, over five metastatic lesions, and EBV DNA concentration above the cutoff value may represent risk factors, whereas RT treatment may be a protective factor. The Kaplan-Meier survival curves also showed an association between RT and improved OS (3-year OS, $27 \%$ vs. $13 \% ; p<0.001$ )

(Fig. 2A). As expected, patients with the aforementioned risk factors had shorter OS than other patients $(\mathrm{p}<0.001 \mathrm{for}$ all) (Fig. 2B-D). 
Table 2

Multivariable analysis for patients prognosis

\begin{tabular}{|c|c|c|c|}
\hline Characteristic & Hazard ratio & $95 \% \mathrm{Cl}$ & Pvalue \\
\hline \multicolumn{4}{|l|}{ Age (years) } \\
\hline$\leq 47$ & Reference & & \\
\hline$>47$ & 1.183 & $0.929-1.507$ & 0.172 \\
\hline \multicolumn{4}{|l|}{ Gender } \\
\hline Male & Reference & & \\
\hline Female & 0.927 & $0.669-1.285$ & 0.650 \\
\hline \multicolumn{4}{|l|}{ Smoking } \\
\hline No smoking & Reference & & \\
\hline Smoking & 1.160 & $0.903-1.489$ & 0.246 \\
\hline \multicolumn{4}{|l|}{ Family history } \\
\hline No & Reference & & \\
\hline Yes & 0.746 & $0.469-1.187$ & 0.217 \\
\hline \multicolumn{4}{|l|}{ T stage } \\
\hline $\mathrm{T}_{1}-\mathrm{T}_{2}$ & Reference & & \\
\hline $\mathrm{T}_{3}-\mathrm{T}_{4}$ & 0.940 & $0.686-1.289$ & 0.702 \\
\hline \multicolumn{4}{|l|}{ N stage } \\
\hline $\mathrm{N}_{0}-\mathrm{N}_{1}$ & Reference & & \\
\hline $\mathrm{N}_{2}-\mathrm{N}_{3}$ & 1.379 & $0.982-1.886$ & 0.056 \\
\hline \multicolumn{4}{|c|}{ No. of metastatic organs } \\
\hline 1 & Reference & & \\
\hline$>1$ & 1.897 & $1.401-2.568$ & $<0.001$ \\
\hline \multicolumn{4}{|c|}{ No. of metastatic lesions } \\
\hline$\leq 5$ & Reference & & \\
\hline$>5$ & 2.246 & $1.670-3.020$ & $<0.001$ \\
\hline \multicolumn{4}{|c|}{ EBV-DNA(Copies/ml) } \\
\hline$\leq 25,000$ & Reference & & \\
\hline$>25,000$ & 1.479 & $1.132-1.930$ & 0.004 \\
\hline \multicolumn{4}{|c|}{ Chemotherapy regimens } \\
\hline TPF & Reference & & \\
\hline TP & 0.799 & $0.560-1.141$ & 0.218 \\
\hline PF & 0.835 & $0.580-1.203$ & 0.334 \\
\hline GP & 0.881 & $0.619-1.254$ & 0.481 \\
\hline Others & 0.821 & $0.460-1.464$ & 0.504 \\
\hline Radiotherapy & & & \\
\hline
\end{tabular}




\begin{tabular}{|llll|}
\hline Characteristic & Hazard ratio & $\mathbf{9 5 \%} \mathrm{Cl}$ & $P$ value \\
\hline Non-RT & Reference & & \\
\hline RT & 0.665 & $0.511-0.864$ & 0.002 \\
\hline
\end{tabular}

\section{Clinical characteristics of patients that did or did not underwent RT in different risk stratifications}

According to the risk factors defined in the previous subsection, we divided patients into eight subgroups: group A, single organ metastasis, EBV DNA concentration $\leq 25,000$ copies/ $\mathrm{ml}$, and 5 or fewer metastatic lesions; group B, single organ metastasis, EBV DNA concentration $>25,000$ copies $/ \mathrm{ml}$, and 5 or fewer metastatic lesions; group C, multiple organs metastasis, EBV DNA concentration $\leq$ 25,000 copies/ml, and 5 or fewer metastatic lesions; group D, multiple organs metastasis, EBV DNA concentration $>25,000 \mathrm{copies} / \mathrm{ml}$, and 5 or fewer metastatic lesions; group E, single organ metastasis, EBV DNA concentration $\leq 25,000 \mathrm{copies} / \mathrm{ml}$, and more than 5 metastatic lesions; group F, single organ metastasis, EBV DNA concentration > 25,000 copies/ml, and more than 5 metastatic lesions; group G, multiple organs metastasis, EBV DNA concentration $\leq 25,000$ copies $/ \mathrm{ml}$, and more than 5 metastatic lesions; and group $H$, multiple organs metastasis, EBV DNA concentration $>25,000$ copies $/ \mathrm{ml}$, and more than metastatic lesions.

The Kaplan-Meier survival curves showed that patients in groups C-H had shorter OS than those in groups A-B; moreover, the OS of group A was significantly longer than that of group $\mathrm{B}(p<0.05$ for all). However, further paired comparisons revealed no significant differences in OS among groups $\mathrm{C}-\mathrm{H}$ ( $p>0.05$ for all) (Fig. 3A). Subsequently, we classified group A as a low-risk subgroup (single organ metastasis, EBV DNA concentration $\leq 25,000$ copies/ml, and 5 or fewer metastatic lesions), group B as an intermediate-risk subgroup (single organ metastasis, EBV DNA concentration $>25,000 \mathrm{copies} / \mathrm{ml}$, and 5 or fewer metastatic lesions), and groups $\mathrm{C}$-H as a high-risk subgroup (multiple organs metastasis or more than 5 metastatic lesions or both). The survival curves of patients in different risk strata are displayed in Fig. 3B. According to the Pearson $\chi^{2}$ test, the subgroups only differed in chemotherapy regimens $(p<0.001, p=0.004$ in low-risk and high-risk subgroups respectively) (Table 3 ) and no significant difference was found in other clinical characteristics. 
Table 3

The clinical characteristics of the patients that did RT and did not do RT in different risk stratifications.

\begin{tabular}{|c|c|c|c|c|c|c|c|c|c|}
\hline \multirow{2}{*}{ Characteristic } & \multicolumn{3}{|l|}{ Low risk } & \multicolumn{3}{|c|}{ Intermediate risk } & \multicolumn{3}{|l|}{ High risk } \\
\hline & non-RT & RT & $\begin{array}{l}P \\
\text { value }\end{array}$ & non-RT & $\mathrm{RT}$ & $\begin{array}{l}P \\
\text { value }\end{array}$ & non-RT & RT & $\begin{array}{l}\mathrm{P} \\
\text { value }\end{array}$ \\
\hline \multicolumn{10}{|l|}{ Age (years) } \\
\hline$\leq 47$ & $15(44.1 \%)$ & $69(52.3 \%)$ & 0.445 & $20(41.7 \%)$ & $42(46.7 \%)$ & 0.595 & $52(49.5 \%)$ & $48(53.5 \%)$ & 0.567 \\
\hline$>47$ & $19(55.9 \%)$ & $63(47.7 \%)$ & & $28(58.3 \%)$ & $48(53.3 \%)$ & & $53(50.5 \%)$ & $41(46.1 \%)$ & \\
\hline \multicolumn{10}{|l|}{ Gender } \\
\hline Male & $28(82.4 \%)$ & 110(83.3\%) & 1.000 & $34(70.8 \%)$ & $75(83.3 \%)$ & 0.124 & $93(88.6 \%)$ & 74(83.1\%) & 0.304 \\
\hline Female & $6(17.6 \%)$ & $22(16.7 \%)$ & & $14(29.2 \%)$ & $15(16.7 \%)$ & & $12(11.4 \%)$ & $15(16.9 \%)$ & \\
\hline \multicolumn{10}{|l|}{ Smoking } \\
\hline No smoking & $22(64.7 \%)$ & $70(53.0 \%)$ & 0.250 & $28(58.3 \%)$ & $53(58.9 \%)$ & 1.000 & $59(56.2 \%)$ & $46(51.7 \%)$ & 0.565 \\
\hline Smoking & $12(35.3 \%)$ & $62(47.0 \%)$ & & $20(41.7 \%)$ & $37(41.1 \%)$ & & $46(43.8 \%)$ & $43(48.3 \%)$ & \\
\hline \multicolumn{10}{|l|}{ Family history } \\
\hline No & $31(91.2 \%)$ & $113(85.6 \%)$ & 0.425 & $44(91.7 \%)$ & $83(92.2 \%)$ & 1.000 & $95(90.5 \%)$ & $81(91.0 \%)$ & 1.000 \\
\hline Yes & $3(8.8 \%)$ & $19(14.4 \%)$ & & $4(8.3 \%)$ & $7(7.8 \%)$ & & $10(9.5 \%)$ & $8(9.0 \%)$ & \\
\hline \multicolumn{10}{|l|}{ T stage \# } \\
\hline $\mathrm{T}_{1}-\mathrm{T}_{2}$ & $4(11.8 \%)$ & $24(18.2 \%)$ & 0.451 & $9(18.8 \%)$ & $17(18.9 \%)$ & 1.000 & $16(15.2 \%)$ & $13(14.6 \%)$ & 1.000 \\
\hline $\mathrm{T}_{3}-\mathrm{T}_{4}$ & $30(88.2 \%)$ & 108(81.8\%) & & $39(81.3 \%)$ & 73(81.1\%) & & $89(84.8 \%)$ & $76(85.4 \%)$ & \\
\hline \multicolumn{10}{|l|}{ N stage \# } \\
\hline $\mathrm{N}_{0}-\mathrm{N}_{1}$ & $9(26.5 \%)$ & $35(26.5)$ & 1.000 & $6(12.5 \%)$ & $18(20.0 \%)$ & 0.348 & $15(14.3 \%)$ & $20(22.5 \%)$ & 0.189 \\
\hline $\mathrm{N}_{2}-\mathrm{N}_{3}$ & $25(73.5 \%)$ & 132(73.5\%) & & $42(87.5 \%)$ & $72(80.0 \%)$ & & $90(85.7 \%)$ & $69(77.5 \%)$ & \\
\hline \multicolumn{10}{|l|}{$\begin{array}{l}\text { No. of } \\
\text { metastatic } \\
\text { organ }\end{array}$} \\
\hline 1 & $34(100.0 \%)$ & $132(100.0 \%)$ & - & $48(100.0 \%)$ & $90(100.0 \%)$ & - & $34(32.4)$ & $37(41.6 \%)$ & 0.232 \\
\hline$>1$ & - & - & & - & - & & $71(67.6 \%)$ & $52(58.4 \%)$ & \\
\hline \multicolumn{10}{|l|}{$\begin{array}{l}\text { No. of } \\
\text { metastatic } \\
\text { tumor number }\end{array}$} \\
\hline$\leq 5$ & $34(100.0 \%)$ & $132(100.0 \%)$ & - & $48(100.0 \%)$ & $90(100.0 \%)$ & - & $15(14.3 \%)$ & $19(21.3 \%)$ & 0.256 \\
\hline$>5$ & - & - & & - & - & & $90(85.7 \%)$ & $70(78.7 \%)$ & \\
\hline \multicolumn{10}{|c|}{$\begin{array}{l}\text { EBV- } \\
\text { DNA(Copies/ml) }\end{array}$} \\
\hline$\leq 25,000$ & $34(100.0 \%)$ & $132(100.0 \%)$ & - & - & - & - & $25(23.8 \%)$ & $23(25.8 \%)$ & 0.868 \\
\hline$>25,000$ & - & - & & $48(100.0 \%)$ & $90(100.0 \%)$ & & $80(76.2 \%)$ & $66(74.2 \%)$ & \\
\hline \multicolumn{10}{|l|}{$\begin{array}{l}\text { Chemotherapy } \\
\text { regimens }\end{array}$} \\
\hline TPF & $7(20.6 \%)$ & $36(27.3 \%)$ & $\begin{array}{l}< \\
0.001\end{array}$ & $11(22.9 \%)$ & $26(28.9 \%)$ & 0.336 & $22(21.0 \%)$ & $26(29.2 \%)$ & 0.004 \\
\hline
\end{tabular}




\begin{tabular}{|lllllll|}
\hline & Low risk & & \multicolumn{2}{l|}{ Intermediate risk } & \multicolumn{2}{l|}{ High risk } \\
\hline TP & $5(14.7 \%)$ & $36(27.3 \%)$ & $10(20.8 \%)$ & $20(22.2 \%)$ & $21(20.0 \%)$ & $29(32.6 \%)$ \\
\hline PF & $15(44.1 \%)$ & $27(20.5 \%)$ & $10(20.8 \%)$ & $26(28.9 \%)$ & $33(31.4 \%)$ & $18(20.2 \%)$ \\
GP & $5(14.7 \%)$ & $3(2.3 \%)$ & $4(8.3 \%)$ & $6(6.7 \%)$ & $9(8.6 \%)$ & $0(0.0 \%)$ \\
\hline others & $2(5.9 \%)$ & $30(22.7 \%)$ & $13(27.1 \%)$ & $12(13.3 \%)$ & $20(19.0 \%)$ & $16(18.0 \%)$ \\
\hline
\end{tabular}

\section{Patients' outcomes in different risk stratifications}

We further investigated the differences in OS between patients that did and did not receive RT in each classification of risk. Interestingly, we found that not all patients benefited from RT. We found statistical differences in OS among patients in the low-risk and intermediaterisk subgroups ( $p=0.039$ and $p=0.010$, respectively), whereas no significant difference was found in the high-risk subgroup $(p=0.076)$ (Fig. 4). Subsequently, we performed the Cox proportional hazards regression model for all subgroups (Table 4) and found that RT lowered the mortality risk for patients in the low-risk (HR, 0.490; $95 \% \mathrm{Cl}, 0.232-0.960 ; p=0.042)$ and intermediate-risk subgroups (HR, $0.582 ; 95 \% \mathrm{Cl}, 0.357-0.947 ; p=0.029)$; however, it did not affect high-risk patients (HR, 0.718; 95\% Cl, 0.499-1.033; $p=0.074)$.

Regarding these patients, the mortality risk was higher for those who had multiple organs metastasis $(\mathrm{HR}, 1.518 ; 95 \% \mathrm{Cl}, 1.032-2.234$; $p=0.034$ ), whereas the presence of multiple ( $>5$ ) metastatic lesions or a pretreatment EBV DNA copies level above cutoff did not seem to worsen this risk (HR: 1.564, 95\% Cl: 0.955-2.562, $p=0.076$; HR: 1.127, 95\% Cl: 0.745-1.707, $p=0.571$, respectively). 
Table 4

Multivariable analysis for patients prognosis in different risk stratifications.

\begin{tabular}{|c|c|c|c|c|c|c|c|c|c|}
\hline & Low risk & & & Intermec & risk & & High ri & & \\
\hline Characteristic & $\mathrm{HR}$ & $95 \% \mathrm{Cl}$ & $\begin{array}{l}P \\
\text { value }\end{array}$ & $\mathrm{HR}$ & $95 \% \mathrm{Cl}$ & $\begin{array}{l}P \\
\text { value }\end{array}$ & $\mathrm{HR}$ & $95 \% \mathrm{Cl}$ & $\begin{array}{l}P \\
\text { value }\end{array}$ \\
\hline Age (years) & & & & & & & & & \\
\hline$\leq 47$ & Referenc & & & Referenc & & & Referer & & \\
\hline$>47$ & 1.311 & $\begin{array}{l}0.724- \\
2.375\end{array}$ & 0.371 & 1.403 & $\begin{array}{l}0.870- \\
2.262\end{array}$ & 0.165 & 1.051 & $\begin{array}{l}0.745- \\
1.480\end{array}$ & 0.778 \\
\hline Gender & & & & & & & & & \\
\hline Male & Referenc & & & Referenc & & & Referer & & \\
\hline Female & 0.486 & $\begin{array}{l}0211- \\
1.118\end{array}$ & 0.089 & 0.657 & $\begin{array}{l}0.361- \\
1.194\end{array}$ & 0.168 & 1.527 & $\begin{array}{l}0.948- \\
2.461\end{array}$ & 0.082 \\
\hline Smoking & & & & & & & & & \\
\hline No smoking & Referenc & & & Referenc & & & Referer & & \\
\hline Smoking & 1.219 & $\begin{array}{l}0.665- \\
2.235\end{array}$ & 0.522 & 1.130 & $\begin{array}{l}0.693- \\
1.843\end{array}$ & 0.632 & 1.266 & $\begin{array}{l}0.883- \\
1.816\end{array}$ & 0.199 \\
\hline Family history & & & & & & & & & \\
\hline No & Referenc & & & Referenc & & & Referer & & \\
\hline Yes & 0.748 & $\begin{array}{l}0.246- \\
2.278\end{array}$ & 0.609 & 0.462 & $\begin{array}{l}0.157- \\
1.355\end{array}$ & 0.160 & 1.057 & $\begin{array}{l}0.572- \\
1.955\end{array}$ & 0.859 \\
\hline T stage & & & & & & & & & \\
\hline $\mathrm{T}_{1}-\mathrm{T}_{2}$ & Referenc & & & Referenc & & & Referer & & \\
\hline $\mathrm{T}_{3}-\mathrm{T}_{4}$ & 0.723 & $\begin{array}{l}0.349- \\
1.499\end{array}$ & 0.383 & 1.110 & $\begin{array}{l}0.605- \\
2.036\end{array}$ & 0.737 & 0.843 & $\begin{array}{l}0.526- \\
1.349\end{array}$ & 0.475 \\
\hline N stage & & & & & & & & & \\
\hline $\mathrm{N}_{0}-\mathrm{N}_{1}$ & Referenc & & & Referenc & & & Referer & & \\
\hline $\mathrm{N}_{2}-\mathrm{N}_{3}$ & 2.318 & $\begin{array}{l}1.098- \\
4.891\end{array}$ & 0.027 & 1.051 & $\begin{array}{l}0.589- \\
1.875\end{array}$ & 0.867 & 1.367 & $\begin{array}{l}0.860- \\
2.175\end{array}$ & 0.186 \\
\hline $\begin{array}{l}\text { No. of metasta } \\
\text { organs }\end{array}$ & & & & & & & & & \\
\hline 1 & - & & & - & & & Referer & & \\
\hline$>1$ & - & - & - & - & - & - & 1.518 & $\begin{array}{l}1.032- \\
2.234\end{array}$ & 0.034 \\
\hline $\begin{array}{l}\text { No. of metasta } \\
\text { tumor }\end{array}$ & & & & & & & & & \\
\hline$\leq 5$ & - & & & - & & & Referer & & \\
\hline$>5$ & - & - & - & - & - & - & 1.564 & $\begin{array}{l}0.955- \\
2.562\end{array}$ & 0.076 \\
\hline EBV-DNA(Copi & & & & & & & & & \\
\hline$\leq 25,000$ & - & & & - & & & Referer & & \\
\hline$>25,000$ & - & - & - & - & - & - & 1.127 & $\begin{array}{l}0.745- \\
1.707\end{array}$ & 0.571 \\
\hline
\end{tabular}




\begin{tabular}{|c|c|c|c|c|c|c|c|c|c|}
\hline & \multicolumn{3}{|c|}{ Low risk } & \multicolumn{3}{|c|}{ Intermediate risk } & \multicolumn{3}{|c|}{ High risk } \\
\hline \multicolumn{10}{|c|}{$\begin{array}{l}\text { Chemotherapy } \\
\text { regimens }\end{array}$} \\
\hline TPF & \multicolumn{3}{|c|}{ Reference } & \multicolumn{3}{|c|}{ Reference } & \multicolumn{3}{|c|}{ Reference } \\
\hline TP & 0.717 & $\begin{array}{l}0.294- \\
1.749\end{array}$ & 0.465 & 0.770 & $\begin{array}{l}0.386- \\
1.537\end{array}$ & 0.459 & 0.818 & $\begin{array}{l}0.500- \\
1.341\end{array}$ & 0.426 \\
\hline PF & 0.814 & $\begin{array}{l}0.336- \\
1.971\end{array}$ & 0.648 & 0.619 & $\begin{array}{l}0.295- \\
1.300\end{array}$ & 0.205 & 0.962 & $\begin{array}{l}0.584- \\
1.583\end{array}$ & 0.878 \\
\hline GP & 0.922 & $\begin{array}{l}0.379- \\
2.241\end{array}$ & 0.858 & 0.925 & $\begin{array}{l}0.486- \\
1.759\end{array}$ & 0.812 & 0.700 & $\begin{array}{l}0.417- \\
1.174\end{array}$ & 0.176 \\
\hline Others & 1.696 & $\begin{array}{l}0.439- \\
6.550\end{array}$ & 0.444 & 0.859 & $\begin{array}{l}0.316- \\
2.333\end{array}$ & 0.766 & 0.688 & $\begin{array}{l}0.258- \\
1.837\end{array}$ & 0.455 \\
\hline \multicolumn{10}{|c|}{ Radiotherapy } \\
\hline Non-RT & \multicolumn{3}{|c|}{ Reference } & \multicolumn{3}{|c|}{ Reference } & \multicolumn{3}{|c|}{ Reference } \\
\hline RT & 0.490 & $\begin{array}{l}0.232- \\
0.960\end{array}$ & 0.042 & 0.582 & $\begin{array}{l}0.357- \\
0.947\end{array}$ & 0.029 & 0.718 & $\begin{array}{l}0.499- \\
1.033\end{array}$ & 0.074 \\
\hline
\end{tabular}

\section{Discussion}

In this study, we stratified dmNPC patients into different risk levels based on the number of metastatic lesions, number of metastatic organs, and level of pretreatment EBV DNA. In exploring the role of LRRT, we found that patients with a single metastatic organ and no more than five metastases benefited the most from the therapy, and among which, the patients with EBV DNA concentration $\leq 25,000$ copies/ml have better OS than those with EBV DNA concentration $>25,000$ copies/ml, which provides important information for individual treatment management in clinical practice.

Distant metastasis has become the main cause of death for NPC patients $(3,10)$. Among all patients with distant metastasis, some had it detected at initial admission, which is defined as dmNPC (5). Unlike patients with metastasis after treatment, these patients had no previous RT for the primary tumor. Whether the use of LRRT is necessary has become a concern of clinicians. Recently, two studies have shown that the addition of LRRT to PCT is associated with a longer survival time for dmNPC $(6,11)$. However, another question needs to be clarified: can all these patients benefit from RT? By analyzing the role of LRRT in dmNPC, You et al. found that patients with liver metastasis did not benefit from the primary tumor treatment while patients with other metastasis did (12). But the M1 stage subdivisions of the study did not take tumor burden and pre-treatment EBV DNA copies into considerations. Similarly, NCCN guidelines recommend chemotherapy combined with LRRT only for patients with limited metastasis sites or a low tumor burden, but the standard for "low tumor burden" of dmNPC is not defined (7).

In this study, we confirmed that local RT was a protective factor for dmNPC patients. Consistently with previous studies, more than five metastases and multiple metastatic organs were identified as independent risk factors $(13,14)$. The cutoff value for the number of metastases was based on the definition of 'oligo metastasis' used in clinical trials (15). Moreover, it has been demonstrated that pretreatment EBV DNA is closely associated with prognosis in locally advanced NPC $(16,17)$. Similar to non-metastatic patients, our results suggested that high levels of EBV DNA may also be associated with worse prognosis in dmNPC patients. Based on the three identified prognostic factors, we divided all patients into eight groups then further classified these groups into three risk levels according to the statistical differences in survival among the eight groups. Due to limitations of the TNM staging system for metastatic patients, the prognosis of patients in the M1 stage could not be further classified. Based on our results, we stratified these patients into different risk levels to facilitate a general prognosis assessment according to their baseline data.

More importantly, our team was the first to identify the optimal candidates for LRRT based on a biomarker and tumor burden. In exploring the role of LRRT, we surprisingly found that only low- and moderate-risk patients (single metastatic organ and no more than five metastases) benefited from primary tumor treatment. This phenomenon may be explained by the following: as the illness of patients with oligo metastases is more likely to be controlled by PCT, they should be responsive to LRRT, which is a good way to prevent 
further disease progression from the primary tumor. However, primary RT may not improve the survival of patients with more than five metastatic lesions or multiple metastatic organs. Considering the high cost and serious treatment-related toxicity, the administration of LRRT should be treated with caution. The main treatment goals for high-risk patients should include long-term survival with tumor and improvement of life quality (18). Therefore, systemic chemotherapy and symptomatic treatment may be preferred treatment strategies for these patients.

The continuous progress of medical science has improved the OS of dmNPC patients remarkably. However, as shown in this study, the survival condition of high-risk patients is still unsatisfactory, with a 3-year survival rate of $35 \%$. Therefore, new therapeutic methods need to be developed, such as epidermal growth factor receptor (EGFR) targeted drugs. Unfortunately, although EGFR overexpression was detected in NPC, a retrospective study showed that the use of anti-EGFR drugs did not further improve the survival of dmNPC patients $(19,20)$. Immunotherapy, represented by PD-1 antibodies treatment, is another recent promising research path (21). To explore the treatment efficacy of PD-1 antibody in metastatic NPC, our group launched a global multicenter, double-blind, randomized controlled phase III clinical trial. At present, patients' enrollment has been completed and we are looking forward to the results of long-term followup.

Our study has the following limitations: our study design was retrospective and selective bias was unavoidable. Furthermore, we conducted a single center study and most cases came from epidemic areas. Therefore, our conclusions need to be confirmed by multicenter prospective clinical trials.

\section{Declarations}

All methods were carried out in accordance with relevant guidelines and regulations as the National Comprehensive Cancer Network (NCCN) guidelines and eighth edition of the American Joint Committee on Cancer staging system.

\section{Availability of data and materials}

The datasets used and/or analyzed during the current study are available from the corresponding author on reasonable request. If anyone wants to request the data from the study, please contact maihq@sysucc.org.cn.

\section{Acknowledgements}

This work was supported by grants from the National Key R\&D Program of China (2017YFC1309003ه2017YFC0908500), the National Natural Science Foundation of China (No. 81425018, No. 81672868, No. 81602371هNo81802775), the Sci-Tech Project Foundation of Guangzhou City (201707020039), the Sun Yat-sen University Clinical Research 5010 Program, the Special Support Plan of Guangdong Province (No. 2014TX01R145), the Natural Science Foundation of Guangdong Province (No. 2017A030312003, No.2017A030312003), the Sci-Tech Project Foundation of Guangdong Province (No. 2014A020212103), the Health \& Medical Collaborative Innovation Project of Guangzhou City (No. 201400000001, No.201803040003), the Planned Science and Technology Project of Guangdong Province (2019B020230002), the National Science \& Technology Pillar Program during the Twelfth Five-year Plan Period (No. 2014BAI09B10),the PhD Start-up Fund of Natural Science Foundation of Guangdong Province (2016A030310221), the cultivation foundation for the junior teachers in Sun Yat-sen University(16ykpy28), and the Fundamental Research Funds for the Central Universities.

\section{Funding}

This study was supported by grants from the National Key R\&D Program of China (2017YFC1309003)

\section{Author contributions}

L-QT, H-QM and Q-YC carried out the study concepts. J-HY, X-SS, B-BX, L-TL, S-SG, J-DL and G-DJ collected patient materials and patient follow-up information. J-HY, X-SS, B-BX, L-TL, S-SG, J-DL and G-DJ participated in the data analysis/interpretation. J-HY, X-SS and B-BX participated in quality control of data and algorithms. J-HY, X-SS, B-BX and L-TL performed statistical analyses. J-HY, X-SS and B-BX participated in manuscript editing. L-QT, Q-YC and H-QM coordinated the study and critically revised the manuscript. All authors read and approved the final manuscript.

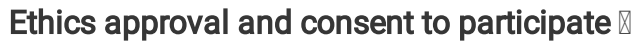


This retrospective study was approved by the Clinical Research Committee of Sun Yat Sen University Cancer Center. Patients were required to provide written informed consent before enrolling in the study.

\section{Consent for publication}

Not applicable.

\section{Competing interests}

The authors declare no competing interests

\section{References}

1. Chua MLK, Wee JTS, Hui EP, Chan ATC. Nasopharyngeal carcinoma. The Lancet. 2016;387(10022):1012-24.

2. Chen YP, Chan ATC, Le QT, Blanchard P, Sun Y, Ma J. Nasopharyngeal carcinoma. Lancet (London, England). 2019;394(10192):6480.

3. Sun XS, Liu SL, Luo MJ, Li XY, Chen QY, Guo SS, et al. The Association Between the Development of Radiation Therapy, Image Technology, and Chemotherapy, and the Survival of Patients With Nasopharyngeal Carcinoma: A Cohort Study From 1990 to 2012. International journal of radiation oncology, biology, physics. 2019;105(3):581-90.

4. Wei WI, Sham JS. Nasopharyngeal carcinoma. Lancet (London, England). 2005;365(9476):2041-54.

5. Lee AW, Ng WT, Chan YH, Sze H, Chan C, Lam TH. The battle against nasopharyngeal cancer. Radiotherapy and oncology: journal of the European Society for Therapeutic Radiology and Oncology. 2012;104(3):272-8.

6. Rusthoven CG, Lanning RM, Jones BL, Amini A, Koshy M, Sher DJ, et al. Metastatic nasopharyngeal carcinoma: Patterns of care and survival for patients receiving chemotherapy with and without local radiotherapy. Radiotherapy and Oncology. 2017;124(1):139-46.

7. Pfister DG, Spencer S, Adelstein D, Adkins D, Anzai Y, Brizel DM, et al. Head and Neck Cancers, Version 2.2020, NCCN Clinical Practice Guidelines in Oncology. J Natl Compr Canc Netw. 2020;18(7):873-98.

8. Sun X, Chen W, Liu S, Liang Y, Chen Q, Guo S, et al. Individualized concurrent chemotherapy by pretreatment plasma Epstein-Barr viral DNA in II-III stage nasopharyngeal carcinoma: A propensity score matching analysis using a large cohort. Cancer medicine. 2019;8(9):4214-25.

9. Li Y, Chen QY, Tang LQ, Liu LT, Guo SS, Guo L, et al. Concurrent chemoradiotherapy with or without cetuximab for stage II to IVb nasopharyngeal carcinoma: a case-control study. BMC cancer. 2017;17(1):567.

10. Zhang M, Li J, Shen G, Zou X, Xu J, Jiang R, et al. Intensity-modulated radiotherapy prolongs the survival of patients with nasopharyngeal carcinoma compared with conventional two-dimensional radiotherapy: A 10-year experience with a large cohort and long follow-up. Eur J Cancer. 2015;51(17):2587-95.

11. Chen MY, Jiang R, Guo L, Zou X, Liu Q, Sun R, et al. Locoregional radiotherapy in patients with distant metastases of nasopharyngeal carcinoma at diagnosis. Chinese journal of cancer. 2013;32(11):604-13.

12. Zou X, You R, Liu H, He YX, Xie GF, Xie ZH, et al. Establishment and validation of M1 stage subdivisions for de novo metastatic nasopharyngeal carcinoma to better predict prognosis and guide treatment. European journal of cancer (Oxford, England: 1990). 2017;77:117 - 26.

13. Sun XS, Liang YJ, Liu SL, Li XY, Chen QY, Guo SS, et al. Establishment and validation of a nomogram for predicting survival in patients with de novo metastatic nasopharyngeal carcinoma. Oral oncology. 2019;94:73-9.

14. Tatinati S, Veluvolu KC, Sun-Mog H, Nazarpour K. Real-time prediction of respiratory motion traces for radiotherapy with ensemble learning. Conference proceedings: Annual International Conference of the IEEE Engineering in Medicine and Biology Society IEEE Engineering in Medicine and Biology Society Annual Conference. 2014;2014:4204-7.

15. Palma DA, Olson R, Harrow S, Gaede S, Louie AV, Haasbeek C, et al. Stereotactic ablative radiotherapy versus standard of care palliative treatment in patients with oligometastatic cancers (SABR-COMET): a randomised, phase 2, open-label trial. Lancet (London, England). 2019;393(10185):2051-8.

16. Chan AT, Lo YM, Zee B, Chan LY, Ma BB, Leung SF, et al. Plasma Epstein-Barr virus DNA and residual disease after radiotherapy for undifferentiated nasopharyngeal carcinoma. Journal of the National Cancer Institute. 2002;94(21):1614-9. 
17. Lin JC, Wang WY, Chen KY, Wei YH, Liang WM, Jan JS, et al. Quantification of plasma Epstein-Barr virus DNA in patients with advanced nasopharyngeal carcinoma. The New England journal of medicine. 2004;350(24):2461-70.

18. Trilling GM, Cho H, Ugas MA, Saeed S, Katunda A, Jerjes W, et al. Spinal metastasis in head and neck cancer. Head \& neck oncology. 2012;4:36.

19. Leong JL, Loh KS, Putti TC, Goh BC, Tan LK. Epidermal growth factor receptor in undifferentiated carcinoma of the nasopharynx. The Laryngoscope. 2004;114(1):153-7.

20. Sun XS, Liang YJ, Li XY, Liu SL, Chen QY, Tang LQ, et al. Palliative chemotherapy with or without anti-EGFR therapy for de novo metastatic nasopharyngeal carcinoma: a propensity score-matching study. Drug design, development and therapy. 2019;13:320716.

21. Reck M, Rodríguez-Abreu D, Robinson AG, Hui R, Csőszi T, Fülöp A, et al. Pembrolizumab versus Chemotherapy for PD-L1-Positive Non-Small-Cell Lung Cancer. The New England journal of medicine. 2016;375(19):1823-33.

\section{Figures}

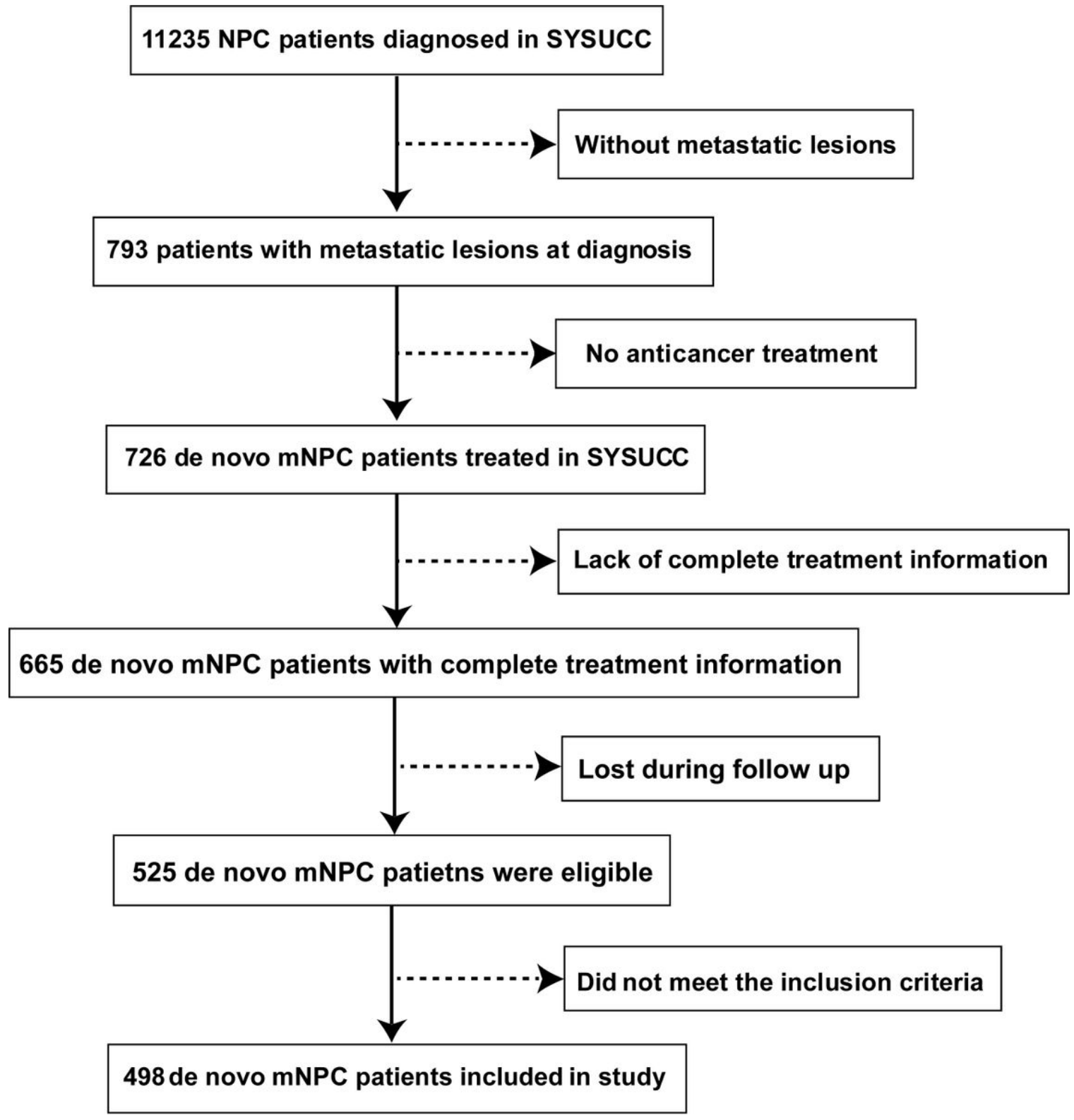

Figure 1

Flow chart showing patients enrollment in study cohort. 
A
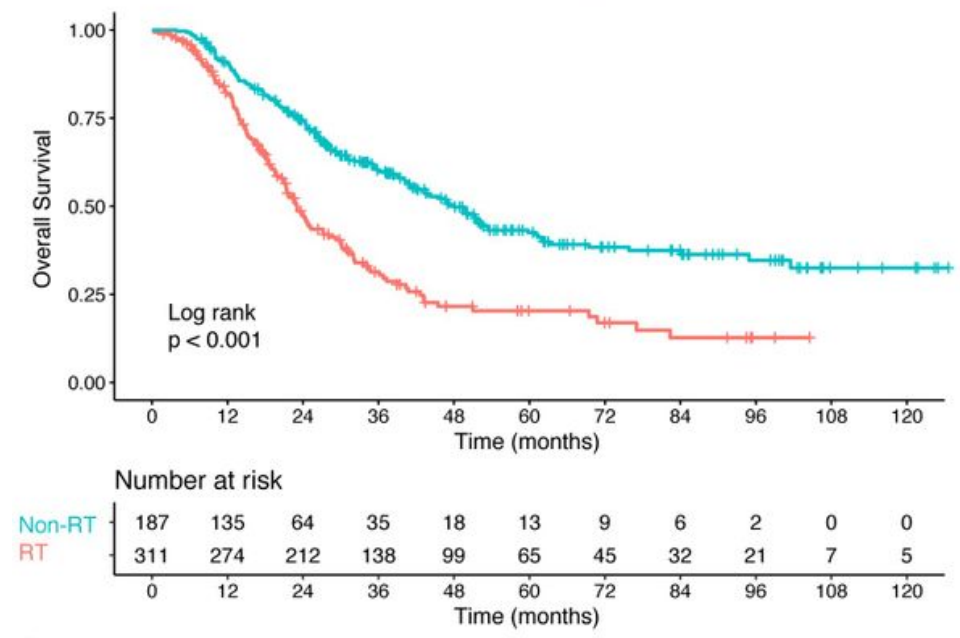

C Strata $+\leq 5$ sites $+>5$ sites

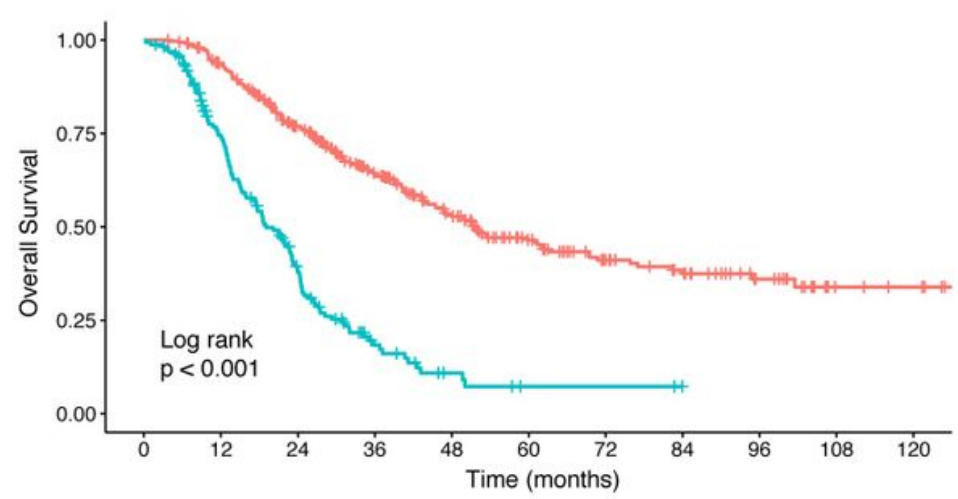

Number at risk

$>5$ sites \begin{tabular}{ccccccccccc}
338 & 304 & 227 & 157 & 111 & 76 & 52 & 38 & 23 & 7 & 5 \\
160 & 105 & 49 & 16 & 6 & 2 & 2 & 0 & 0 & 0 & 0 \\
\hline 0 & 12 & 24 & 36 & 48 & 60 & 72 & 84 & 96 & 108 & 120 \\
Time (months) &
\end{tabular}
B Strata + EBV $\leq 25000+E B V>25000$

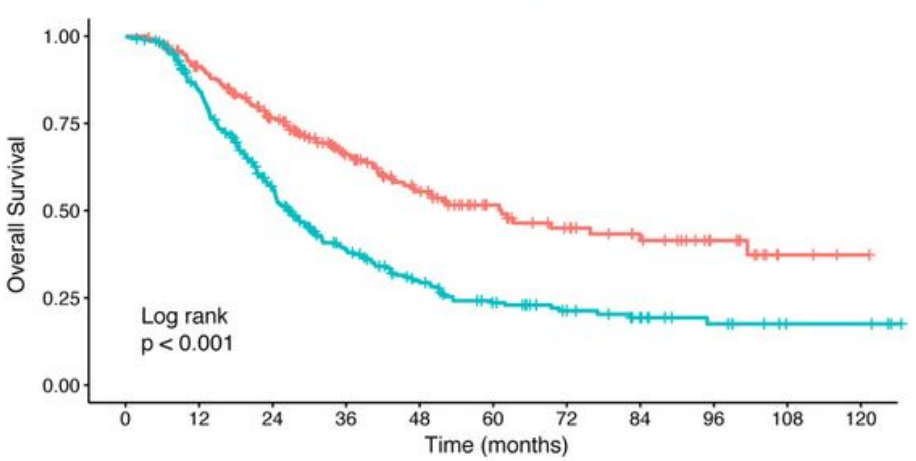

Number at risk
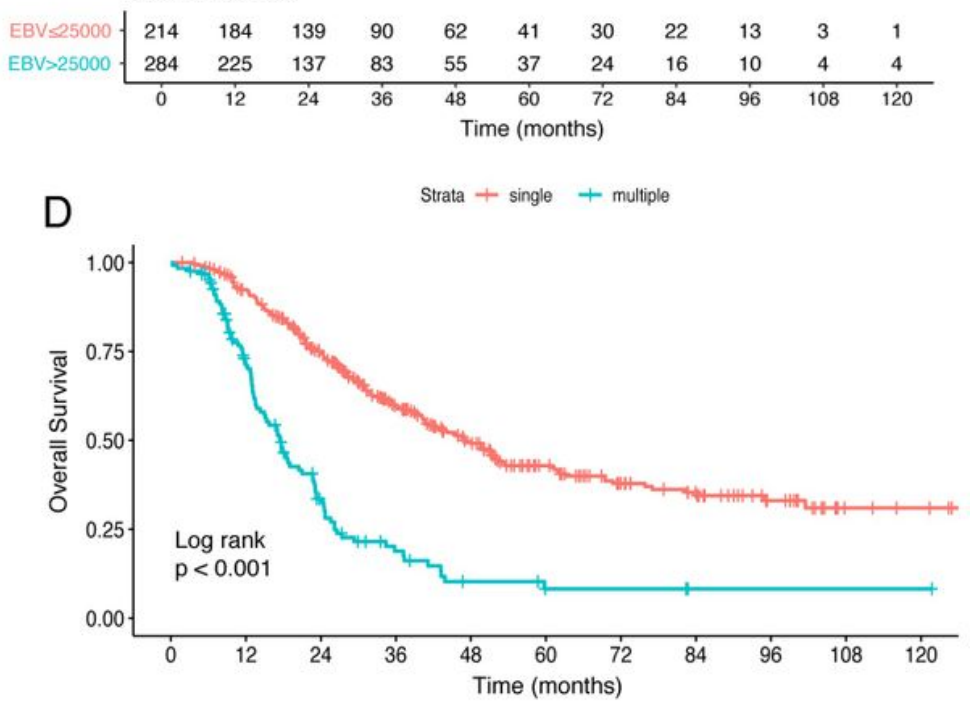

Number at risk

single \begin{tabular}{ccccccccccc}
375 & 333 & 245 & 159 & 111 & 75 & 51 & 37 & 22 & 6 & 4 \\
123 & 76 & 31 & 14 & 6 & 3 & 3 & 1 & 1 & 1 & 1 \\
\hline 0 & 12 & 24 & 36 & $\begin{array}{c}48 \\
12\end{array}$ & $\begin{array}{c}60 \\
\text { Time (months) }\end{array}$ & 72 & 84 & 96 & 108 & 120
\end{tabular}

\section{Figure 2}

Kaplan-Meier survival curves for the factors that may influence survival outcomes in the training cohort. Radiotherapy (A), Epstein-Barr virus DNA copies (B), numbers of metastatic lesions (C), numbers of metastatic organs (D). 
A $\quad$ Strata + Group $\mathrm{A}+$ Group $\mathrm{C}+$ Group $\mathrm{E}+$ Group $\mathrm{G}$
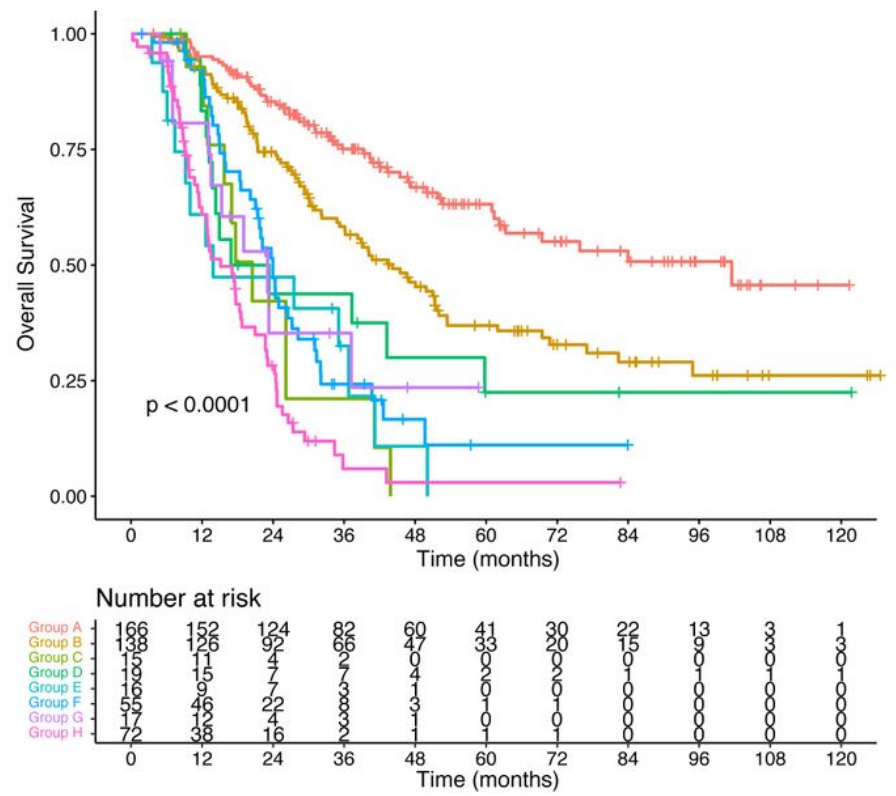

B Strata + Low risk + Intermediate risk + High risk
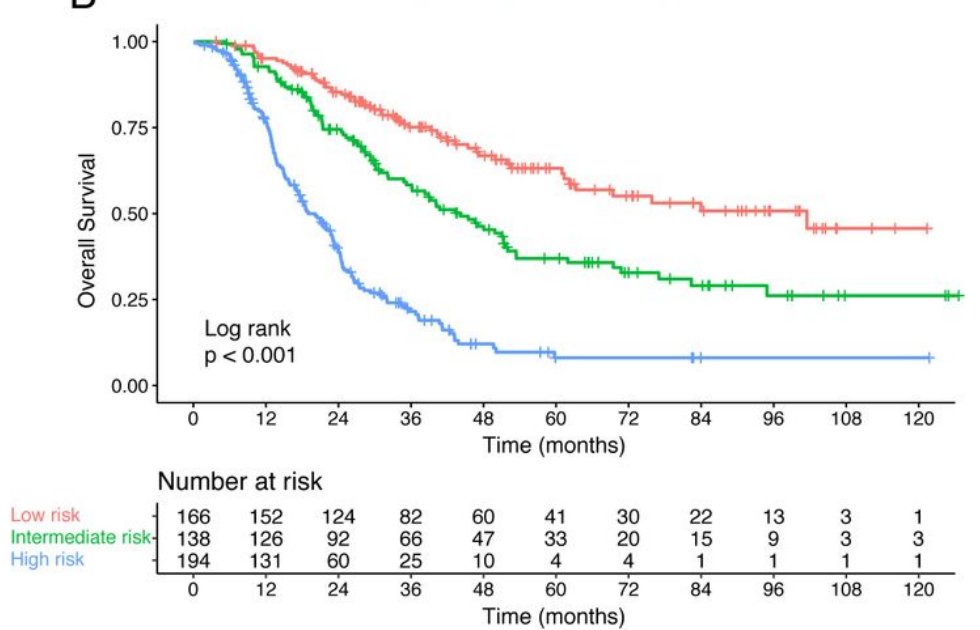

Figure 3

Kaplan-Meier survival curves for the risk factors (A) and different risk stratifications (B). 
A

Strata + Non-RT + RT
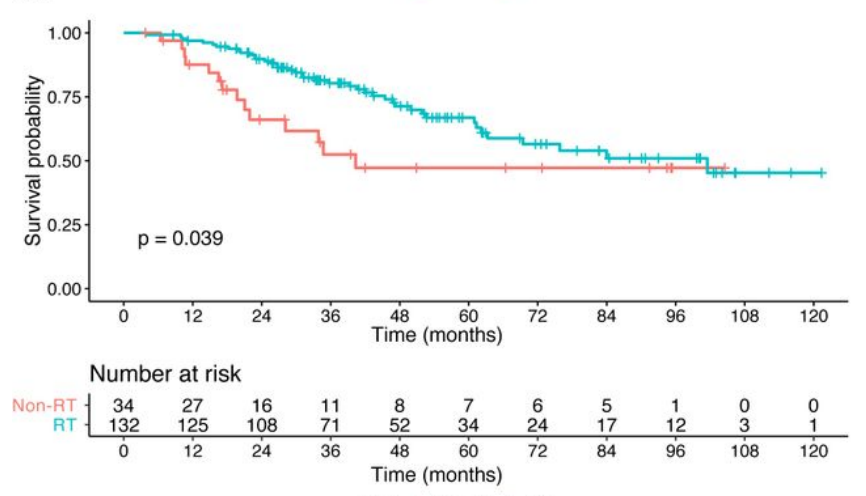

\section{B}
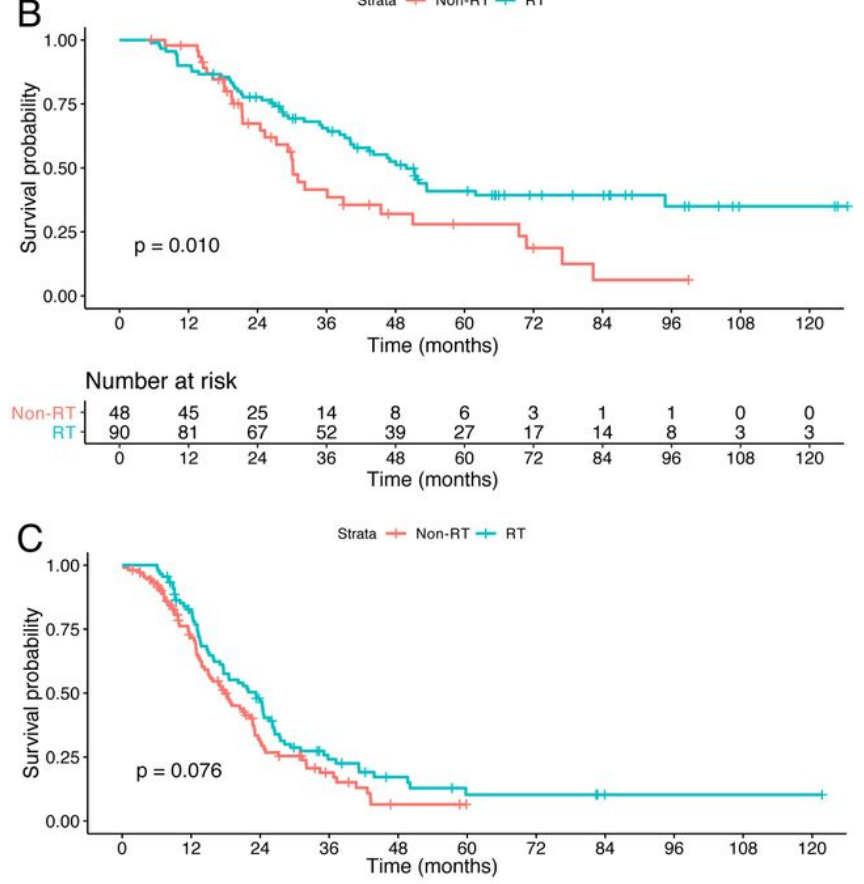

Number at risk

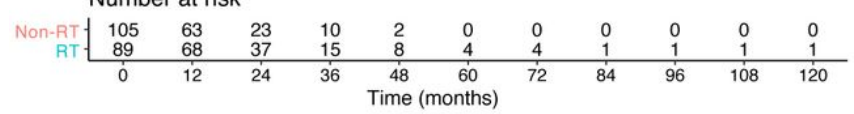

Figure 4

Comparison of overall survival of patients in the locoregional radiotherapy (LRRT) and non-LRRT groups: low-risk patients (A), intermediate-risk patients (B) and high-risk patients (C). 